\title{
$1 \lambda$ Ronchi tester to obtain the wave front aberration in converging optical systems using phase shifting interferometry
}

\section{Probador de Ronchi con precisión de $1 \lambda$ en las aberraciones del frente de onda, para sistemas ópticos convergentes usando la interferometria de desplazamiento de fase}

\author{
J. Sánchez-Paredes(1), G. Silva-Ortigoza(2), J. Castro-Ramos ${ }^{(1, *)}$, and José Sasian(3) \\ 1. Instituto Nacional de Astrofísica Óptica y Electrónica, apartado postal 51 y 216, Tonantzintla, \\ Puebla, C.P. 72000, México. \\ 2. Facultad de Ciencias Físico Matemáticas de la Benemérita Universidad Autónoma de Puebla, \\ Apartado Postal 1152, 72001, Puebla, México. \\ 3. College of Optical Sciences, The University of Arizona, 1630 E. University Blvd., P.O. Box 210094, \\ Tucson, AZ 85721-0094, USA. \\ ${ }^{*}$ E) Email: jcastro@inaoep.mx \\ Recibido / Received: 22/10/2012. Aceptado / Accepted: 07/11/2012. \\ DOI: http://dx.doi.org/10.7149/OPA.45.4.461
}

\begin{abstract}
:
We implement a device to verify in a quantitative way the quality of any converging optical system by making an improvement to the source illumination of the Ronchi test and by implementing the phase shifting interferometry technique (PSI). We describe the design, implementation and verification of this specific device which has the capability to obtain the transversal aberration in two perpendicular directions. Illumination source was a set of LEDs powered with constant current to eliminate variations in the irradiance collected by the camera (CCD), and varying the voltage to increase the intensity of each different wavelength LED we test surfaces with different roughness. The PSI technique was developed by giving movements to the Ronchi ruling with step motors. The device has the capability to test any converging optical systems of either small or large f numbers, and working distances until $5000 \mathrm{~mm}$. Aberrated wavefront with a precision of $1 \lambda$ was computed when the light source is off axis for any rotationally symmetric mirror.
\end{abstract}

Key words: Ronchi Tester, Sensitivity, Phase Shifting.

\section{RESUMEN:}

Implementamos un dispositivo para verificar cuantitativamente la calidad de cualquier sistema óptico convergente, haciendo una mejora a la fuente de iluminación de la prueba de Ronchi e implementando la técnica de interferometria de desplazamiento de fase (PSI). Describimos el diseño, la implementación y verificación de este dispositivo especifico el cual tiene la capacidad de obtener la aberración transversal en 2 direcciones perpendiculares. Como fuente de iluminación se usó un conjunto de LEDs alimentados con corriente constante para eliminar variaciones en la irradiancia captada por la cámara (CCD), al variar el voltaje incrementamos la intensidad del LED (cada uno de longitud de onda diferente) lo que permite probar superficies con diferentes rugosidades. La técnica PSI fue implementada dando movimientos a la rejilla de Ronchi con un motor a pasos. El dispositivo tiene la capacidad de probar cualquier sistema óptico convergente sin importar si tiene un $f / \#$ grande o pequeño, y distancias de trabajo de hasta $5000 \mathrm{~mm}$. Se calcula el frente de onda aberrado con una precisión de $1 \lambda$ cuando la fuente de iluminación está fuera del eje óptico para un espejo con simetría de rotación.

Palabras clave: Probador de Ronchi, Sensibilidad, Desplazamiento de Fase. 


\section{REFERENCIAS Y ENLACES / REFERENCES AND LINKS}

[1]. V. Ronchi, "Forty years of history of a grating interferometer", Appl. Opt. 3, 437-451 (1964).

[2]. I. Adachi, "Quantitative measurement of aberration by Ronchi test", Atti Fond. Giorgio Ronchi, Contrib. $1^{\text {st }}$. Naz Ottica 15, 550 (1960).

[3]. A. Cornejo, D. Malacara, "Ronchi test of aspherical surfaces, analysis and accuracy", Appl. Opt. 9, 1897-1901 (1970).

[4]. T. Yatagai, "Fringe scanning Ronchi test for aspherical surfaces", Appl. Opt. 23, 3676-3679 (1984).

[5]. K. Omura, T. Yatagai, "Phase measuring Ronchi test", Appl. Opt. 27, 523-528 (1988).

[6]. H.-J. Lee, "Precision profile measurement of aspheric surfaces by improved Ronchi test", Opt. Eng. 38, 1041-1047 (1999).

[7]. K. Hibino, D. I. Farrant, B. K. Ward, B. F. Oreb, "Dynamic range of Ronchi test with a phase-shifted sinusoidal grating”, Appl. Opt. 36, 6178-6189 (1997).

[8]. J. Castro-Ramos, J. Sasian, "Automatic phase shifting Ronchi tester with a square Ronchi ruling”, Proc. SPIE 5532, 199-210 (2004).

[9]. J. M. Nuñez-Alfonso, A. Cordero-Davila, S. Vergara-Limon, J. Cuautle-Cortes, "Improved Ronchi tester", Appl. Opt. 40, 501-505 (2001).

[10]. J. Arasa, S. Royo, N. Tomas, "Simple method for improving the sampling in profile measurements by use of the Ronchi test", Appl. Opt. 39, 4529-4534 (2000).

[11]. W. S. Meyers, H. P. Stahl, “Contouring of a free oil surface”, Proc. SPIE 1755, 84-94 (1993).

[12]. W. S. Meyers, H. P. Stahl, "Sensitivity of two-channel Ronchi test to grating misalignment", Proc. SPIE 1994, 90-101 (1994).

[13]. D. J. Fischer, H. P. Stahl, “A vector formulation for Ronchi shear surface fitting”, Proc. SPIE 1755, 228238 (1992).

[14]. J. L. Rayces, "Exact Relation between wave aberration and ray aberration”, J. Mod. Opt. 11, 85-88, (1964).

[15]. J. H. Brunning, D. R. Herriott, J. E. Gallagher, D. P. Rosenfeld, A.D. White, D. J. Brangaccio, "Digital wavefront measuring interferometer for testing optical surfaces and lenses", Appl. Opt. 13, 26932703 (1974).

[16]. P. Hariharan, B. F. Oreb, T. Eiju, “Digital phase shifting interferometry: A simple error-compensating phase calculation algorithm", Appl. Opt. 26, 2504-2506 (1987).

[17]. R. Mukundam, K. Ramakrishnan, Moment Functions in Image Analysis, pp. 9-37, 127-128, World Scientific Publishing Co. Pte. Ltd, Singapore (1998).

[18]. J. E. Greivenkamp, J. H. Bruning, "Phase shifting interferometry", Chapter 14 in Optical Shop Testing, D. Malacara, Edt., Wiley \& Sons, NY, USA (1992).

[19]. D. C. Ghiglia, L. A. Romero, "Robust two-dimensional weighted and unweigthed phase unwrapping that uses fast transforms and iterative methods", J. Opt. Soc. Am. A 11, 107-117 (1994).

[20]. R. Cubalchini, "Modal wave-front phase derivative measurements", J. Opt. Soc. Am. 69, 972-977 (1979).

[21]. F. Roddier, C. Roddier, "Wavefront reconstruction using iterative Fourier transforms”, Appl. Opt. 30, 1325-1327 (1991).

[22]. R. Burden, D. Faires, Numerical Analysis, Thomson Brooks, USA (2005).

[23]. J. Wyant, http://www.optics.arizona.edu/jcwyant/ Zernikes/ZernikeEquations .htm.

[24]. A. Cordero-Davila, A. Cornejo-Rodriguez, O. Cardona-Nuñez, "Ronchi and Hartmann test with the same mathematical theory", Appl. Opt. 31, 2370-2376 (1992).

[25]. W. Edwards Deming, Statistical Adjustment of Data, pp. 37-39, John Wiley \& Sons, Inc. (1946).

[26]. B. Cabrera-Perez, J. Castro-Ramos, G. Gordiano-Alvarado, S. Vazquez-Montiel, "Error analysis in a device to test optical systems by using Ronchi test and phase shifting", AIP Conference Proceedings, 992, 858-863 (2008). 


\section{Introduction}

Since Vasco Ronchi [1] developed in 1923 the Ronchi test, it has been used in a qualitative way more than quantitative. Adachi [2] realized a treatment analyzing the sensitivity of the Ronchi test in a quantitative manner. Cornejo and Malacara [3] gave the accuracy of the Ronchi test when any rotationally symmetric mirror was under test, and showed the precision or accuracy that can be obtained with the Ronchi test. Yatagai [4] proposed a quantitative Ronchi test by using a phase synchronous detection technique. Omura and Yatagai [5] published a method to test lenses using the Ronchi's method as a shearing interferometer. Lee, Ho-Jae [6] used a laser as an illumination source and a holographic sinusoidal grating. The dynamic range of a phase-shifted Ronchi test with an interferometrically recorded sinusoidal grating, was analyzed by Hibino et al [7]. Castro and Sasian [8] used phase shifting interferometry and one square Ronchi ruling to provide quantitative results. Nuñez-Alfonso et al [9] developed an improved Ronchi tester, which uses a LED as the illumination source. Joseph Arasa [10] developed a technique based on multiple ronchigrams. With the purpose to contour dynamic oil surfaces, two channel Ronchi test with an accuracy of 5\% was proposed by Meyers [11]. Errors introduced by different rotational and translation misalignments of the grating in a two Ronchi channel, were analyzed by Meyers [12].

By employing linear algebra vector of the ronchigram sampling and surface fitting together with Gram-Schmidt process Fischer[13] made a data processing procedure to extract the wavefront surface profile.

Despite the many years had passed and the many articles that have been written, there is not in the market a Ronchi tester to be able to recover automatically and quantitatively the wavefront with a precision of one wavelength and the ability to test fast or slow surfaces with different roughness in an almost instantaneous way.

In this paper we described an electro-optomechanical system to analyze the wave front of any converging optical system, which uses conventional LEDs with an electronic circuit to control the current to avoid variations in the intensity and to increase or diminish the radiant flux. We present the design, implementation and verification of a specific hardware configuration, we use three phase synchronous motors to achieve phase changes in two perpendicular directions; two were used to obtain the total transversal aberrations and the other for defocusing the Ronchi patterns. In section two we present the fundamental concepts of the Ronchi test and the phase shifting interferometry technique, also we describe the method used to obtain the wrapping phase and we discuss the problem of solving the valid phase pixels employing geometrical moments together with a method to choose the threshold of the images to be processed. In section three the experimental setup is described. Section four describes the sensitivity of our device when any symmetrical mirror is tested and a $1 \lambda$ experimental setup to obtain the wavefront aberration is discussed. In section five two examples are shown; one mirror and one lens. Finally conclusions are given.

\section{Fundamental concepts}

In the exit pupil plane of the system under the Ronchi test as shown Fig. 1, the wavefront aberration is represented as $W(x, y)$. The ray proceeded from the point $(x, y)$ in the exit pupil and intersecting the paraxial focal plane at the point $\left(T A_{x}, T A_{y}\right)$ is related according to Rayces [14] in good approximation as

$$
\frac{\partial W}{\partial x}=-\frac{T A_{x}}{R}, \frac{\partial W}{\partial y}=-\frac{T A_{y}}{R},
$$

where $R$ is the radius of curvature of the wavefront. When the Ronchi grating is placed at the plane perpendicular to the optical axis and its grating lines are parallel to the $y$-axis, by considering the grating to have a sinusoidal density profile we can obtain the Ronchigrams given by back projecting the Ronchi grating on the exit pupil of the optical system under test. In the case of synchronous phase detection, the phase of the Ronchi grating is change stepwise $\delta_{n}=n d / N,(n=0,1,2,3 \ldots N-1)$, where $d$, $\delta$, and $\gamma$ denote the grating pitch, initial phase term of the grating, and fringe contrast, 
respectively; the positions of the fringe peaks for $\delta=0$ and the derivative of the wavefront aberration is given by $[4,5]$

$$
I(x, \delta)=1+\gamma \cos \left[\frac{2 \pi}{d}\left(R \frac{\partial W}{\partial y}-\delta\right)\right] .
$$

Hence the total change of phase is equal to the period of the grating; $N$ denotes the number of steps between the pitch of the ruling. Therefore we obtain $N$ Ronchigrams with different phases. According to the principle of synchronous phase detection as Brunning [15] explained, the summations with proper weights

$$
\begin{aligned}
& C_{1}(x)=\sum_{n=0}^{N-1} I\left(x_{n}, \delta_{n}\right) \cos \left(\frac{2 \pi n}{N}\right), \\
& S_{1}(x)=\sum_{n=0}^{N-1} I\left(x_{n}, \delta_{n}\right) \sin \left(\frac{2 \pi n}{N}\right),
\end{aligned}
$$

are calculated to obtain the phase term of the Ronchigrams for the first derivative of the wave front, thus the relative phase is computed by averaging $N$ Ronchigrams with different phases. Additionally the transversal aberration for $X$ and $Y$ axes (giving a $90^{\circ}$ rotation to the ruling) are calculated with Eq. (4):

$$
T A_{x}=T A_{y}=-\frac{d}{2 \pi} \arctan \left(\frac{S_{1}(x)}{C_{1}(x)}\right) .
$$

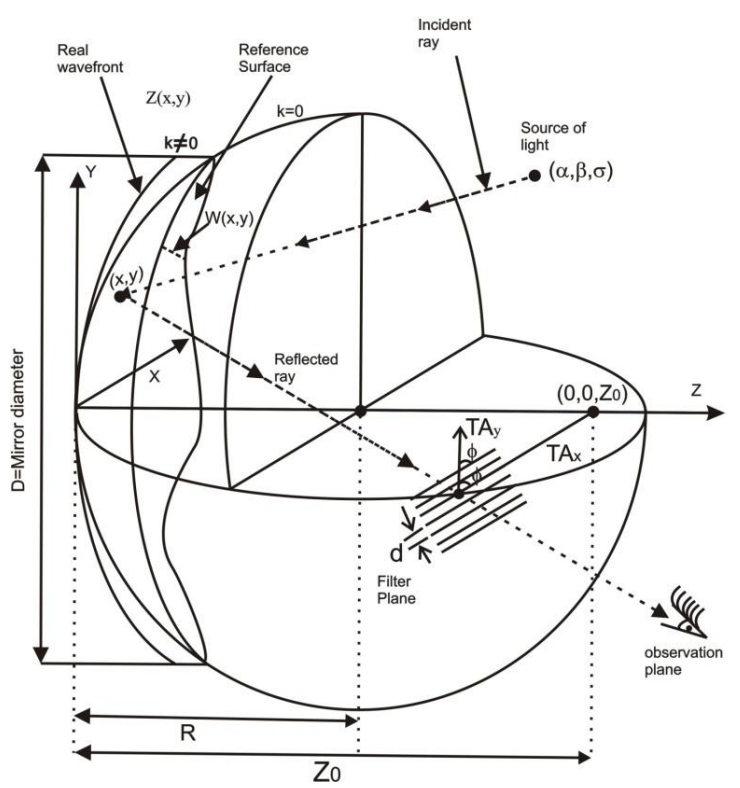

Fig.1. Geometry of the Ronchi test.

\subsection{Phase wrapping}

In order to obtain the phase of the Ronchigrams, according to Hariharan [16] we use five measurements of irradiance $I_{1}, I_{2}, I_{3}, I_{4}, I_{5}$ corresponding to additional phase shifting of $-2 \alpha,-\alpha, 0,+\alpha,+2 \alpha$, respectively with , the phase difference $\varphi$, and the preliminary calibration of the motors to ensure that the phase step $\alpha$ is approximately equal to $90^{\circ}$ can be carried out with the equations:

$$
\begin{aligned}
& \tan [\varphi(x, y)]= \\
& =\frac{2\left[I_{2}(x, y)-I_{4}(x, y)\right]}{2 I_{3}(x, y)-I_{5}(x, y)-I_{2}(x, y)^{\prime}} \\
& \cos \alpha=\frac{I_{5}(x, y)-I_{1}(x, y)}{2\left[I_{2}(x, y)-I_{4}(x, y)\right]} .
\end{aligned}
$$

With the obtained ronchigrams it is possible to compute the wrapped phase of the ronchigrams, but Eq. (5) shows discontinuities that should be removed as is explained in next section.

\subsection{Choosing the boundary valid phase points}

The digitized ronchigrams have true and false phase points, depending on the boundary geometry of the surface under test. Generally this boundary is circular but there are segmented telescopes with hexagonal, annular or square boundaries. There are many methods for differentiating valid from invalid phase pixels included in commercial phase measuring interferometer software but in order to obtain the true phase points for any shape, we developed the phase shifting interferometric technique together with geometrical moments, defined as one base set $\left\{x^{p}, y^{p}\right\}$. The bidimensional orders $(p+q)^{\text {th }}$ are denoted by $m_{p q}$ for one binary image these can be expressed according to Mukundam [17]

$$
m_{p q}=\int_{\tau} x^{p} y^{q} f(x, y) d x d y .
$$

where $p, q=0,1,2,3 \ldots$, and $\tau$ is the space region in pixels in which the intensity function of the image $f(x, y)$ is defined. We employ the boundary moments which consist of boundary points that compose the object. The zero order moment $m_{00}$ represents the total intensity of any image and the first order functions $m_{10}, m_{01}$ give the intensity moments along the $y$ and $x$ 
axes of the image respectively. The centroid $\left(x_{0}, y_{0}\right)$ of the intensities are given by $x_{0}=m_{10} / m_{00}, y_{0}=m_{01} / m_{00}$.

This image centroid was employed as the origin of our reference system. The calculated geometrical moments with respect to their intensity centroid are called central moments and are defined as

$$
\mu_{p q}=\iint_{\tau}\left(x-x_{0}\right)^{p}\left(y-y_{0}\right)^{q} d x d y,
$$

$p, q=0,1,2,3 \ldots$ The orientation angle $\theta$ is one of the principal inertia axes with the $\mathrm{x}$ axis given by

$$
\theta=\frac{1}{2} \tan ^{-1}\left(\frac{2 \mu_{11}}{\mu_{20}-\mu_{02}}\right) .
$$

Equations

$I_{n 1}=\left(\mu_{20}+\mu_{02}\right)+\frac{\sqrt{\left(\mu_{20}+\mu_{02}\right)^{2}+4 \mu_{11}^{2}}}{2}$,

$I_{n 2}=\left(\mu_{20}+\mu_{02}\right)-\frac{\sqrt{\left(\mu_{20}-\mu_{02}\right)^{2}+4 \mu_{11}^{2}}}{2}$,

and $\theta$ can be used to define the ellipse of an image which has the same inertia moments and the principal direction axes as the original image. The $a$ and $b$ radii of the minor and mayor axis of the ellipse are given by

$$
a=2\left(\frac{I_{n 1}}{\mu_{00}}\right)^{1 / 2}, b=2\left(\frac{I_{n 2}}{\mu_{00}}\right)^{1 / 2} .
$$

With this parameter we choose the valid and invalid phase points of the Ronchigram, eliminating the non-useful part of the image as we saw in Fig. 2.

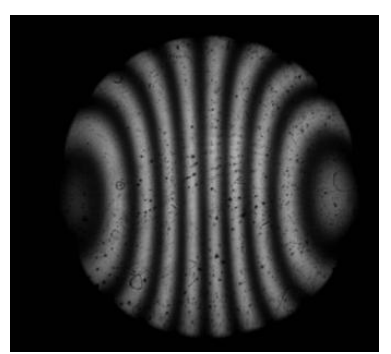

(a)

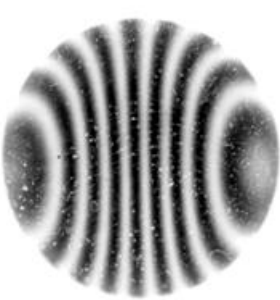

(b)
Fig. 2. Two ronchigrams: (a) before these were processed, (b) after valid phase points were obtained.

\subsection{Phase unwrapping}

When phase shifting interferometry is used we must to correct the discontinuities produced by the arc tangent function (Eq. 5) which is only defined from $-\pi / 2$ to $+\pi / 2$. The first correction is to extend the range from 0 to $2 \pi$. According to Greivenkamp [18] this is possible since the sign of sine and cosine functions are well known independently of the tangent sign. The result is to produce the phase modulus $2 \pi$. According to Ghiglia and Romero [19], we assume that we know the phase $\phi$ modulus $2 \pi$ of any function defined in a discrete grid of points. Given the wrapped phase values $\varphi_{i, j}$, we determined the values of the unwrapped phase $\phi_{i, j}$, we have rearranged the Ghiglia and Romero [19] method to calculate the differences between rows and columns of the wrapped phase, respectively.

\subsection{Wavefront computing}

There are many methods to recover the wavefront of any optical system starting from their transversal aberrations. The Cubalchini or Roddier methods [20,21] are some of them; these are usually more used between others. We prefer to implement the two dimensional Simpson 1/3 method to integrate the transversal aberrations to recover the wavefront aberration, we choose this method because the error is minimal when the integral is calculated according with Burden [22].

\subsection{Zernike polynomial}

The wave front aberration $W(x, y)$ can be expressed in terms of the constructed Zernike polynomial functions so that they are orthogonal on a circular region of radial unit; according to Wyant [23] they are related to more common optical aberrations. Twenty polynomials were chosen which results in:

$$
\begin{gathered}
W(x, y)=a_{3} Z_{3}+a_{5} Z_{5}+a_{6} Z_{6}+a_{7} Z_{7}+ \\
+a_{8} Z_{8}+a_{11} Z_{11}+a_{12} Z_{12}+a_{13} Z_{13}+ \\
+a_{14} Z_{14}+a_{15} Z_{15}+a_{16} Z_{16}+a_{17} Z_{17}+ \\
+a_{18} Z_{18}+a_{19} Z_{19}+a_{20} Z_{20}+a_{21} Z_{21}+ \\
+a_{22} Z_{22}+a_{23} Z_{23}+a_{24} Z_{24}+a_{25} Z_{25}
\end{gathered}
$$

where $a_{i}$ are polynomial coefficients and $Z_{i}$ are Zernike polynomials in the expansion for $W(x, y)$. We start with $Z_{3}$ because according to 
Eqs. (5-11), we should avoid either any singularity or ill conditioned system.

\section{Experimental setup}

The experimental setup is shown in Fig. 3. The arrangement consists of the mirror or lens under test, a Ronchi ruling, an interchangeable revolver of LEDs as source of illumination which (none use additional optical components avoiding some additional aberrations as is commonly implemented [1-18]), one Ronchi grating (located for a mirror near of curvature radius, and for a lens close to the focus), placed on a mount with three stepping motors, which had a 200-steps/rev angular resolution controlled by a computer as we see in Fig. 3 . The mount used has three movements, one in the Xaxis and the other in the Y-axis, to obtain in an independent manner the wave front of the surface under test. The Z-axis was used to defocus the optical system. We can see in Fig. 3, the mount is accompanied by two mitutoyo ${ }^{\circledR}$ micrometers with very smooth motion for precision positioning applications, both available in travel ranges from $4-80 \mathrm{~mm}$ and with axial load capacities from $40-450 \mathrm{~N}$ and a resolution of $1 \mu \mathrm{m}$. Instead of the common source of light, we used one that was on a mount that allows us to change the source of illumination. We use several LEDs, which cover a wavelength range from 450-950 $\mathrm{nm}$ (spectral responses are shown in Fig. 4), all were fed employing a small power supply and a variable resistor to vary voltages, in order to have both the correct current and voltage to increase or reduce the intensity of the LED in use. Ronchigrams were detected with Monochrome CCD camera $640 \times 480$ pixels, and finally a software program to compute transversal and wavefront aberrations was developed and employed to automate the process and the method developed.

\subsection{Power supply for source of light}

Using LEDs that can be controlled in intensity is an advantage when we are testing roughness surfaces, aluminized surfaces, surfaces under the polish step or even with liquid surfaces [11]. The source has the advantage because was designed to correct very well the current that goes to the
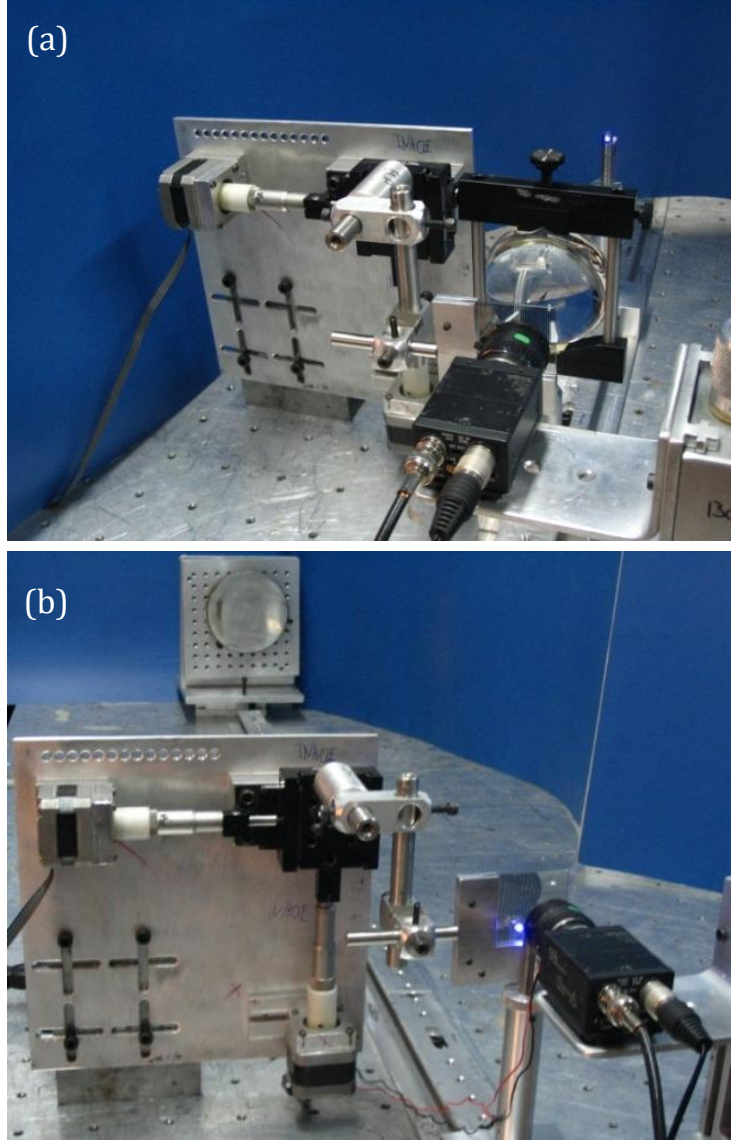

Fig. 3. Ronchi test; experimental setup to test: (a) lenses, (b) mirrors.

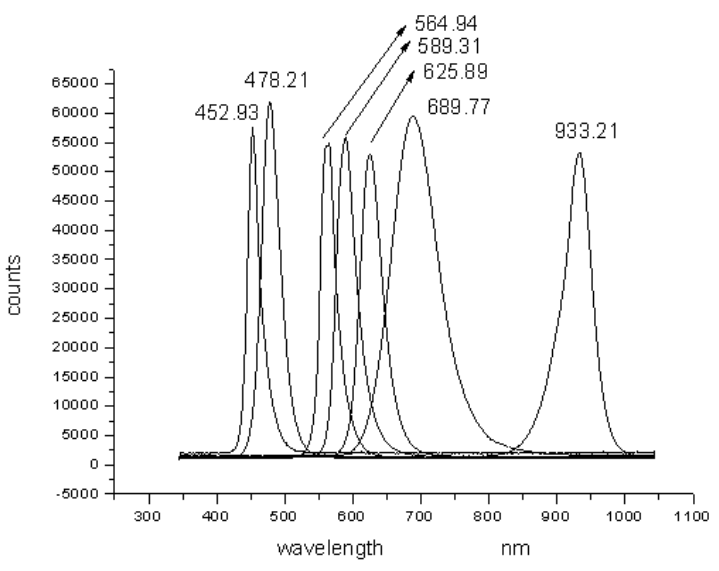

Fig. 4. Spectral peaks of LEDs used as source of illumination.

LED avoiding variations on the intensity. Testing of polished or aluminized surfaces is realized using visible light which gives good results, but in some occasions one should testing roughness surfaces, so that one should use IR wavelength. To carry it one interchange illumination source was used. Spectral radiant flux of several 


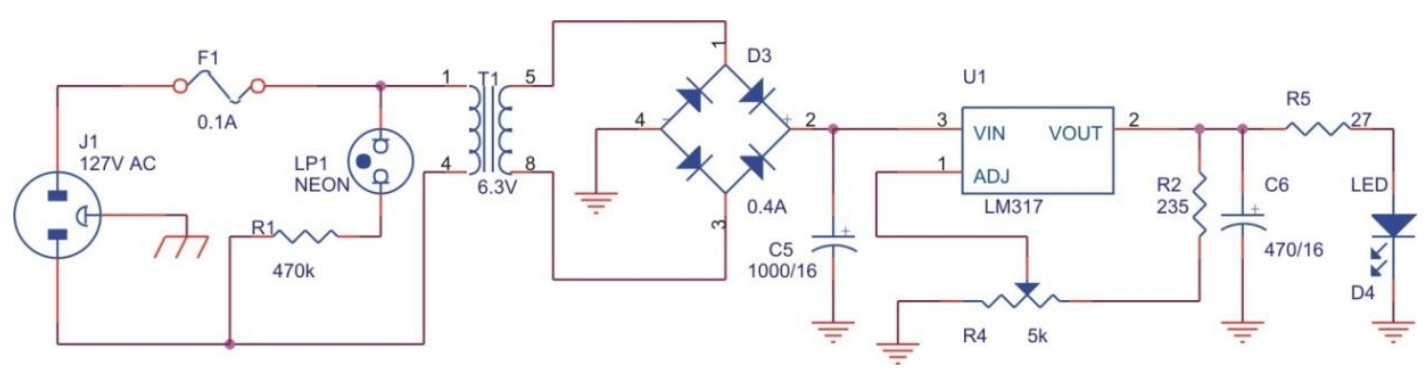

Fig. 5. Layout of the power supply.

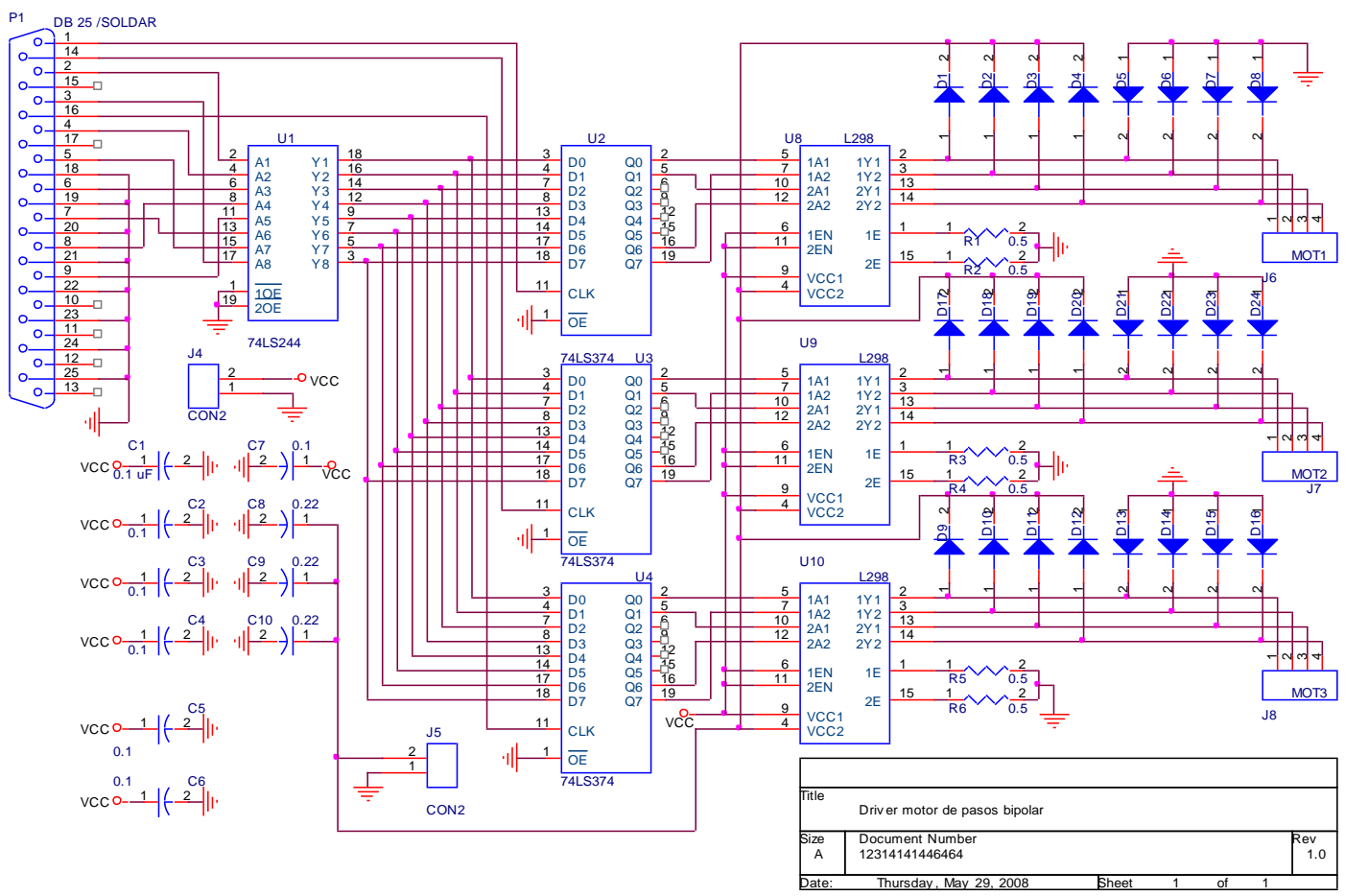

Fig. 6. Schematic diagram, driver of stepping motors.

different wavelength LEDs was measured experimentally. Figure 4 shows the spectral peaks of the LEDs used. Other factor that was analyzed was the $f / \#$, in this case it is important consider the divergence and intensity of the source of light, first of all we calculate the divergence which had a range from 20-140 degrees, greater than one common LED. Each LED was polished close to the cathode, to obtain a better angle of divergence, which also ensured that the illumination was uniform up to a power of $1 \mathrm{~W}$. With these modifications we illuminate totally the area of fast and slow converging optical systems. In order to avoid variations in the irradiance we designed and built a power supply which supplies the LED keeping constant the electrical current. It uses alternating current (ac) and consists of a voltage transformer (T1), a full wave rectifier (D3) and one adjustable regulator (U1) which provides variable voltage to the diode (D4). The irradiance level is carried out by means of R4; this gives power-supply stability in voltaje. Figure 5 shows the layout of the power supply.

\subsection{Electronic circuits to move the motors}

We made an electronic circuit to control three stepping motors; one to move the ruling along the $x$ axis, other to move the ruling along the $y$ axis and the other to control the $z$ axis for defocusing the lens under test. The three motors are bi-polar and were controled via the parallel port of the personal computer. One 74LS244 


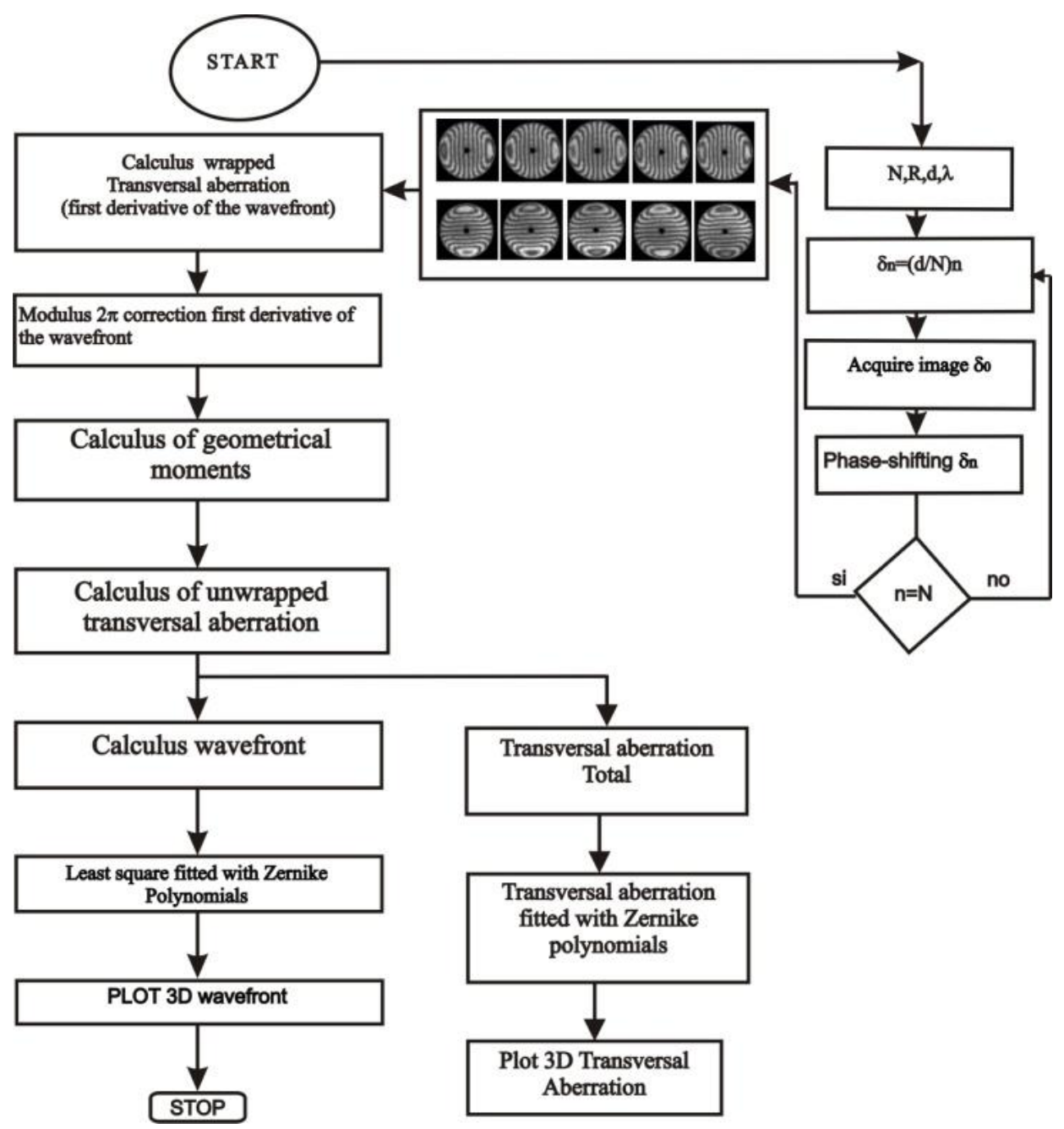

Fig. 7. Flow diagram to calculate simultaneously transversal $X$ and $Y$ aberrations and their wavefront.

buffer was used to re inforce the signal in the data chanel. The control lines of the parallel port include Strobe, Auto feed, and Init. These were useful to register the data in the 74LS374 device. Such data were presented at the L298 bridges of 4 amperes which handle directly the bipolar stepper motors; Figure 6 shows the layout of the circuit.

\subsection{Software}

We combined several programs into one to obtain the wave front aberration. We wrote one routine to move the motor, one routine to capture images, one routine to obtain the transversal aberration, and also we elaborated a program routine to obtain the wavefront aberration. To make the acquisition data, the analysis of the transverse aberration and the wavefront evaluation automatic, we developed a software system of which the flow diagram is shown in Fig. (7).

As the first step, the program requires a set of parameters to acquire Ronchigrams with different reference phases which are stored in a frame grabber memory. In the next step, the phase of the Ronchigram is calculated according to Eq. (4). The calculated phase is wrapped between $-\pi$ and $\pi$ by computation of the trigonometric arctan function, also discontinuities obtained by Eq. (5) were removed. In order to detect true phase points for round shape optical systems, we employ the phase shifting interferometric technique together with geometrical moments calculated according to Eqs. (7-10). Next step, according with Ghiglia 
and Romero, by supposing we know the wrapped phase of any function defined in a discrete grid of points we compute the unwrapped phase. By relatively rotating the surface under test, or by rotating the ruling 900 the $y$-direction derivative of the wave front was measured. Given the unwrapped phase values in both $\mathrm{X}$ and Y-axes, we employed these measurements to fit the coefficients in a transversal aberration expansion of orthogonal functions like Zernike polynomial according with the derivative of Eq. (11). the next step was to plot 3-D the transversal aberration.

Last step, was employ the $1 / 3$ Simpson's rule Burden [17], to obtain the wavefront aberration. In order to realize the fitting of the measured data points of the wave front obtained we used Zernike Polynomials given by Eq. (11). The final step was to plot a graph of the 3-D of the wave front with the computation of their corresponding values of the RMS and PV.

\section{Error in the calculus of the transversal aberration for any mirror}

Although our method works well to check the quality of any converging optical system we developed a method to obtain the sensitivity of our device applied to any symmetrical mirror. After we obtain the derivative of the wave front we proceed to make an error analysis of the transversal aberration for any rotationally symmetric mirror.

From Cordero-Davila et al [24], the transversal aberration components of any reflected ray emerging from any mirror surface $(x, y)$, are given by:

$$
\begin{aligned}
& T A_{x}=x+\left(z_{f}-z\right)\left\{\frac{(x-\alpha)\left(1+z_{x}^{2}+z_{y}^{2}\right)-2 z_{x}\left[z_{y}(y-\beta)+(\gamma-z)\right]}{(\gamma-z)\left(1-z_{x}^{2}-z_{y}^{2}\right)+2\left[z_{x}(x-\alpha)+(y-\alpha)\right]}\right\}, \\
& T A_{y}=y+\left(z_{f}-z\right)\left\{\frac{(y-\beta)\left(1+z_{x}^{2}-z_{y}^{2}\right)-2 z_{y}\left[z_{x}(x-\alpha)+(\gamma-z)\right]}{(\gamma-z)\left(1-z_{x}^{2}-z_{y}^{2}\right)+2\left[z_{x}(x-\alpha)+(y-\alpha)\right]}\right\},
\end{aligned}
$$

where $z_{x}$ and $z_{y}$ are the partial derivatives of sag $z$ (including de conic constant $k$ ) with respect to $x$ and $y$ respectively, and the coordinates of the point light source are $(\alpha, \beta, \gamma)$, see Fig. 1 .

In general the transversal aberration is expressed in terms of the variables involved in the Ronchi test as follows:

$$
T A_{i}\left(\alpha, \beta, \gamma, x, y, z_{f}, k, r\right), \quad i=x, y .
$$

$$
\begin{aligned}
T_{j}=\frac{\partial T_{i}}{\partial j}=f\left(\alpha, \beta, \gamma, x, y, z_{f}, k, r\right), & \\
& j=\alpha, \beta, \gamma, x, y, z_{f}, k, r .
\end{aligned}
$$

For example:

$$
T_{\gamma}=\frac{\partial T_{i}}{\partial \gamma}=\frac{N u m}{D e n}
$$

with:

From Eqs. (12) we can see that:

$$
\begin{aligned}
& \text { Num }=c\left(c^{2} k x^{3}-c^{2} k x^{2} \alpha-\left(c^{2}(k+2) y^{2}-1\right) \alpha+x\left(c^{2} y(k y+2 \beta)-1\right)\right) \times \\
& \quad \times\left(B+c(k+1) z_{f}-1\right)\left(1-c^{2} S^{2}+c^{4} k^{2} S^{2}\left(c^{2} S^{2}-1\right)+k\left(c^{4} S^{4}-3 c^{2} S^{2}+1\right)\right) \\
& \text { Den }=\left(B-1+c^{2}\left((1-2 B-B k) x^{2}+2(k+1) x \alpha+y(y-2 B y-B k y+2 \beta+2 k \beta)\right)+\right. \\
& \left.+c^{4}(k+1) S^{2}\left(k\left(x^{2}-2 x \alpha+y(y-2 \beta)\right)-2(x \alpha+y \beta)-B c(k+1) \gamma+B c^{3}(k+1)(k+2) S^{2} \gamma\right)\right)^{2}
\end{aligned}
$$


where $\quad S^{2}=x^{2}+y^{2}, \quad c=1 / r \quad$ and $\quad B=$ $\sqrt{1-c^{2}(1+k) S^{2}}$.

From Eqs. (15)-(16), the total error according to $[25,26]$ is expressed by

$$
\begin{aligned}
& \Delta T_{i}=\left(\left(\frac{\partial T_{i}}{\partial \alpha} \Delta \alpha\right)^{2}+\left(\frac{\partial T_{i}}{\partial \beta} \Delta \beta\right)^{2}+\right. \\
& +\left(\frac{\partial T_{i}}{\partial \gamma} \Delta \gamma\right)^{2}+\left(\frac{\partial T_{i}}{\partial x} \Delta x\right)^{2}+\left(\frac{\partial T_{i}}{\partial y} \Delta y\right)^{2}+ \\
& \left.+\left(\frac{\partial T_{i}}{\partial k} \Delta k\right)^{2}+\left(\frac{\partial T_{i}}{\partial r} \Delta r\right)^{2}+\left(\frac{\partial T_{i}}{\partial z_{f}} \Delta z_{f}\right)^{2}\right)^{\frac{1}{2}}
\end{aligned}
$$

We employ calipers, one translation stage and manual micrometers to measure the position of the point source, the distance between the vertices of the mirror, the distance to the ruling, and the radius of curvature with a sensitivity of $\Delta \alpha=\Delta \beta=\Delta \gamma=\Delta x=\Delta y=\Delta z_{f}=\Delta r=0.005$ $\mathrm{mm}$.

Coordinates were chosen according to reference [10], when going towards the edge of the optical system aperture, the transverse aberrations reach their maximum. If we use any experimental point, for example if $x=25.92 \pm$ $0.005 \mathrm{~mm}, \quad y=13.39 \pm 0.005 \mathrm{~mm}, \quad r=$ $1378.85 \pm 0.005 \mathrm{~mm}, k=-2.54, \alpha=-1.43 \pm$ $0.005 \mathrm{~mm}, \quad \beta=-8.87 \pm 0.005 \mathrm{~mm}, \quad \gamma=$ $1377.43 \pm 0.005 \mathrm{~mm}, \quad z_{f}=1368.05 \pm 0.0005$ mm, $c=1 / r$, we obtain $\Delta T_{i}=4967.42 \mathrm{~mm}$.

We match the maximun sensitivity of our CCD with a common blue LED which emits from 450 to $500 \mathrm{~nm}$, its maximun is at $478.21 \mathrm{~nm}$ (see figure 4) we obtain the sensitivity of the transversal aberration $\Delta \mathrm{T}_{\mathrm{i}}=10.38 \lambda$, which allow us to calculate the wavefront aberration with a sensitivity of $1 \lambda$.

This sensitivity could be improved using other devices with better resolution for example by using a precision linear stage coupled with high one precision adjustment screw of about 50 $\mathrm{nm}$ from Newport ${ }^{\mathrm{TM}}$, other gratings with different pitch, Cornejo [3] and other wavelength.

\section{Examples}

To show the versatility of our device we test several optical systems such as mirrors made from aluminum alloy 6061, mirrors made with coated or non coated silica glass, also we test biconvex and plane convex lenses during the grinding process. Moreover we checked the optical quality of several microscope and telescope objectives with different f-numbers during the ensemble process. We verify the quality of mirrors with roughness between 5 to 50 microns; depending on the situation we employ the convenient IR LED as source of illumination.

\subsection{Testing a lens}

One first example was to verify a bi convex lens during the polishing process. We tested an $f / \#=1.771$ lens, with diameter of $27.56 \mathrm{~mm}$. We employ the experimental setup shown in Fig. 3a. We compensate the phase error by using Eq. (5) and we use a 25 lines/inch grating and successive displacements of 90 degrees. Figure 8 shows the ronchigrams obtained in $\mathrm{X}$ and $\mathrm{Y}$ directions.

The transversal aberration in the $\mathrm{X}$ axis and the wavefront aberration obtained are shown in Fig. 9, the PV of the transversal aberration in $\mathrm{X}$ axis was $0.59938 \mathrm{~mm}$. The transversal aberration RMS was $0.0923 \mathrm{~mm}$, the phase error was 3.5795 degrees, and the PV OPD was $0.035795 \mathrm{~mm}$. The Zernike coefficients described in section 2.5 are shown in Table I.

\subsection{Testing a mirror}

We tested a mirror with diameter of $99.714 \pm$ $0.005 \mathrm{~mm}$, radius of curvature of $1375.9 \pm 0.005$ $\mathrm{mm}(f / 6.9)$, a conic constant of -2.54 , we compensate the phase error by using Eq. (6) and we use a 25 lines/inch grating with successive displacements of 90 degrees, as shown in Fig. 10.

The transversal aberration in the $\mathrm{X}$ axis and the wave front aberration obtained are shown in Fig. 11, the PV of the transversal aberration in the $\mathrm{X}$ axis was $0.0392413 \mathrm{~mm}$. The Transversal aberration RMS was $0.00411 \mathrm{~mm}$, the phase error was 4.9291 degrees and the PV OPD was $0.0025488 \mathrm{~mm}$. The Zernike coefficients described in section 2.5 are shown in Table II. 

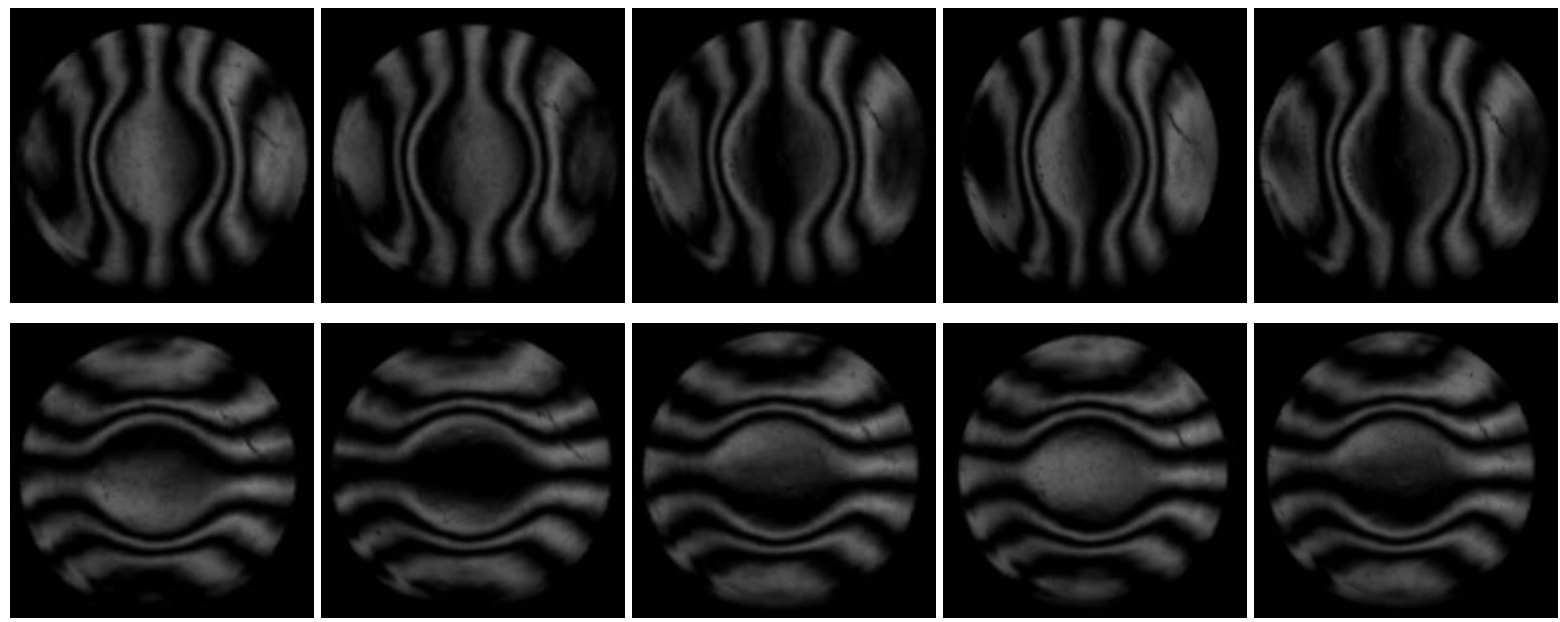

Fig. 8. Ronchigram images with phase shifting of 90 degrees between each one.

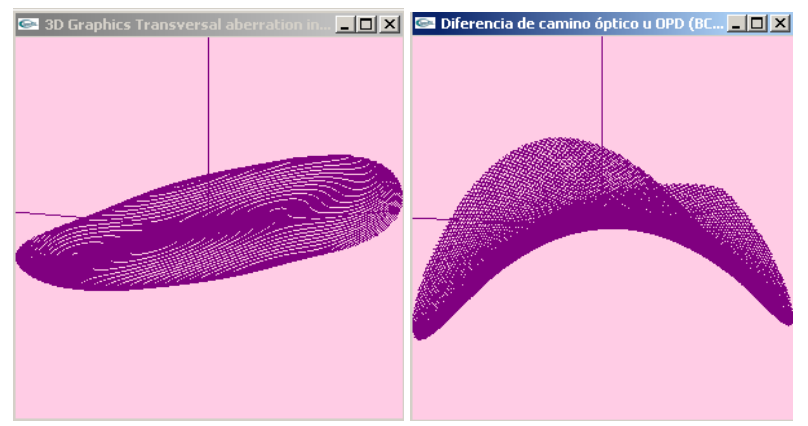

Fig. 9. (a) Transverse aberration in X-axis, (b) Wave front aberration.

Table I

Coefficients values of the lens under test.

\begin{tabular}{|c|c|c|c|c|c|}
\hline \multicolumn{2}{|c|}{$\begin{array}{l}\text { Transversal aberration } \\
\text { coefficients in X ( } \mathrm{mm})\end{array}$} & \multicolumn{2}{|c|}{$\begin{array}{l}\text { Transversal aberration } \\
\text { coefficients in } Y(\mathrm{~mm})\end{array}$} & \multicolumn{2}{|c|}{$\begin{array}{l}\text { Wave front coefficients in } X, Y \\
(\mathrm{~mm})\end{array}$} \\
\hline & -0.1434 & & -0.1171 & & -0.01302 \\
\hline & 1.52862 & $\mathbf{a}_{5}=$ & 1.56314 & $\mathbf{a}_{5}=$ & 0.154058 \\
\hline$a_{6}=$ & 0.0528 & $a_{6}=$ & $7.47255 \times 10^{-3}$ & $a_{6}=$ & 0.0481 \\
\hline $\mathbf{a}_{7}=$ & -0.1919 & $\mathbf{a}_{7}=$ & 0.0188 & $\mathbf{a}_{7}=$ & -0.0102 \\
\hline$a_{8}=$ & 0.1184 & $a_{8}=$ & 0.0465 & $a_{8}=$ & 0.0326 \\
\hline $\mathbf{a}_{11}=$ & -0.1761 & $\mathbf{a}_{11}=$ & 0.0235 & $\mathbf{a}_{11}=$ & $-4.00769 \times 10^{-4}$ \\
\hline $\mathbf{a}_{12}=$ & 0.692 & $\mathbf{a}_{12}=$ & 0.7783 & $\mathbf{a}_{12}=$ & 0.07993 \\
\hline $\mathbf{a}_{13}=$ & -0.072 & $\mathbf{a}_{13}=$ & -0.1965 & $a_{13}=$ & -0.07663 \\
\hline $\mathbf{a}_{14}=$ & 0.2615 & $\mathbf{a}_{14}=$ & $3.20712 \times 10^{-3}$ & $\mathbf{a}_{14}=$ & $7.52675 \times 10^{-3}$ \\
\hline $\mathbf{a}_{15}=$ & 0.0156 & $\mathbf{a}_{15}=$ & -0.0314 & $\mathbf{a}_{15}=$ & -0.01165 \\
\hline $\mathbf{a}_{16}=$ & 0.1444 & $\mathbf{a}_{16}=$ & $-4.21816 \times 10^{-3}$ & $a_{16}=$ & -0.03731 \\
\hline $\mathbf{a}_{17}=$ & 0.1741 & $a_{17}=$ & -0.0872 & $\mathbf{a}_{17}=$ & $-4.13692 \times 10^{-3}$ \\
\hline $\mathbf{a}_{18}=$ & 0.072 & $\mathbf{a}_{18}=$ & -0.0341 & $a_{18}=$ & 0.01349 \\
\hline$a_{19}=$ & $-7.32069 \times 10^{-3}$ & $\mathbf{a}_{19}=$ & 0.0788 & $a_{19}=$ & 0.04362 \\
\hline $\mathbf{a}_{20}=$ & -0.0402 & $\mathbf{a}_{20}=$ & -0.0255 & $\mathbf{a}_{20}=$ & $-8.02781 \times 10^{-4}$ \\
\hline $\mathbf{a}_{21}=$ & -0.6691 & $\mathbf{a}_{21}=$ & -0.6572 & $\mathbf{a}_{21}=$ & -0.06626 \\
\hline $\mathbf{a}_{22}=$ & 0.0219 & $\mathbf{a}_{22}=$ & 0.0611 & $a_{22}=$ & 0.03151 \\
\hline $\mathbf{a}_{23}=$ & -0.183 & $\mathbf{a}_{23}=$ & -0.0149 & $\mathbf{a}_{23}=$ & -0.04072 \\
\hline & 0.0132 & $\mathbf{a}_{24}=$ & 0.011 & $\mathbf{a}_{24}=$ & 0.01226 \\
\hline $\mathbf{a}_{25}=$ & -0.1241 & $\mathbf{a}_{25}=$ & 0.0725 & $\mathbf{a}_{25}=$ & 0.02783 \\
\hline
\end{tabular}



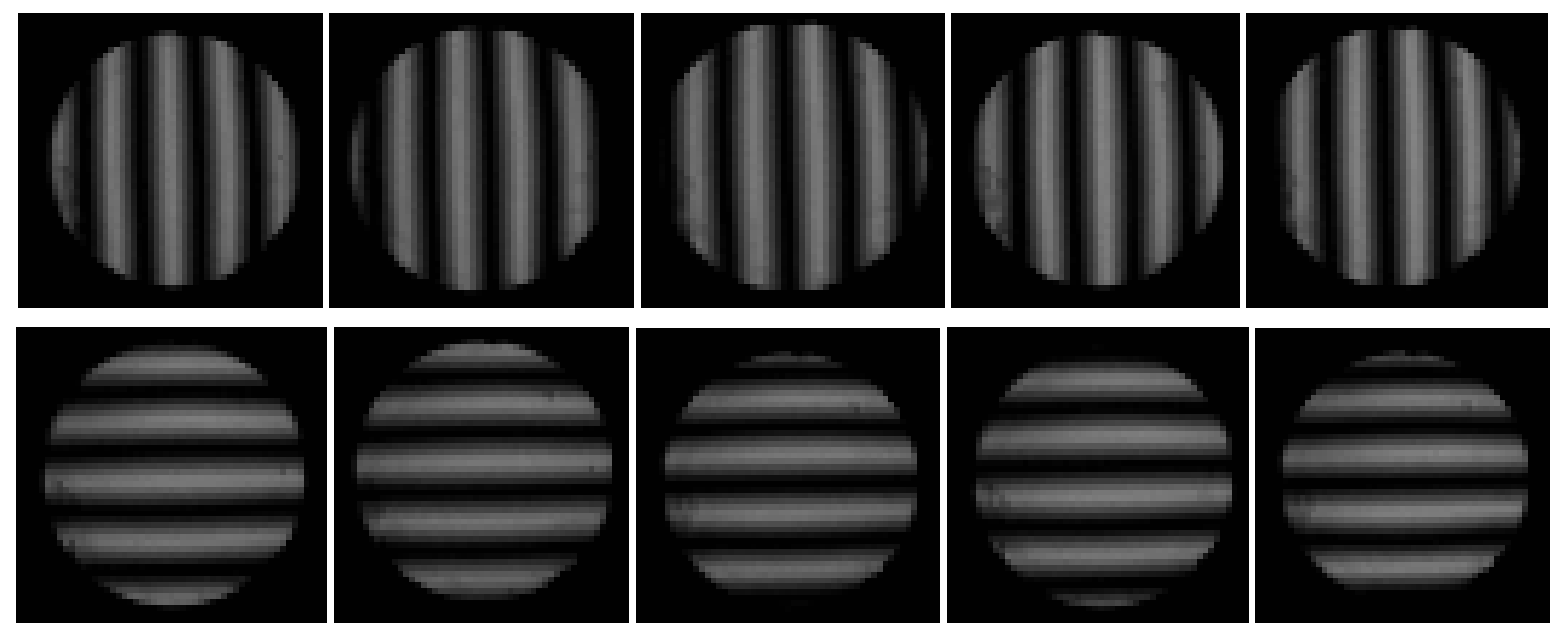

Fig. 10. Ronchigram images with phase shifting of 90 degrees between each one.

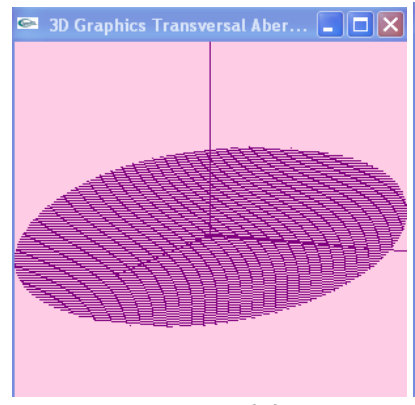

(a)

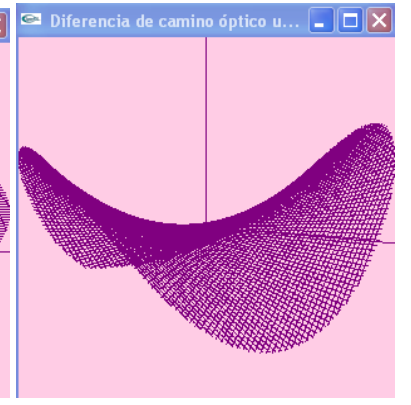

(b)

Fig. 11. (a) Transverse aberration in X-axis, (b) Wave front aberration.

Table II

Coefficients values of the mirror under test.

\begin{tabular}{|c|c|c|c|c|c|}
\hline \multicolumn{2}{|c|}{$\begin{array}{l}\text { Transversal aberration } \\
\text { coefficients in X (mm) }\end{array}$} & \multicolumn{2}{|c|}{$\begin{array}{l}\text { Transversal aberration } \\
\text { coefficients in } \mathrm{Y}(\mathrm{mm})\end{array}$} & \multicolumn{2}{|c|}{$\begin{array}{l}\text { Wave front coefficients in } X, Y \\
(\mathrm{~mm})\end{array}$} \\
\hline & 0.01082 & & -0.0991 & & $4.56748 \times 10^{-4}$ \\
\hline & -0.99982 & & -0.99092 & $\mathbf{a}_{5}=$ & -0.099189 \\
\hline$a_{6}=$ & -0.0838 & $\mathbf{a}_{6}=$ & -0.0773 & $a_{6}=$ & -0.00827 \\
\hline$a_{7}=$ & -0.0471 & & -0.01195 & $\mathbf{a}_{7}=$ & -0.0112 \\
\hline$a_{8}=$ & -0.02686 & $a_{8}=$ & 0.0416 & $a_{8}=$ & $1.01259 \times 10^{-4}$ \\
\hline$a_{11}=$ & 0.05418 & $\mathbf{a}_{11}=$ & 0.08387 & $\mathbf{a}_{11}=$ & 0.04961 \\
\hline$a_{12}=$ & -0.0996 & $\mathbf{a}_{12}=$ & -0.08551 & $\mathbf{a}_{12}=$ & -0.0126 \\
\hline$a_{13}=$ & -0.0284 & $a_{13}=$ & $6.21985 \times 10^{-4}$ & $a_{13}=$ & $-3.12571 \times 10^{-4}$ \\
\hline $\mathbf{a}_{14}=$ & -0.0216 & $\mathbf{a}_{14}=$ & 0.01129 & $\mathbf{a}_{14}=$ & 0.00129 \\
\hline$a_{15}=$ & $-2.8022 \times 10^{-4}$ & $\mathbf{a}_{15}=$ & $2.33838 \times 10^{-4}$ & $\mathbf{a}_{15}=$ & $-7.58314 \times 10^{-5}$ \\
\hline$a_{16}=$ & -0.05269 & $\mathbf{a}_{16}=$ & 0.06265 & $a_{16}=$ & $-3.70289 \times 10^{-4}$ \\
\hline$a_{17}=$ & -0.059 & $a_{17}=$ & 0.07411 & $a_{17}=$ & $-1.19449 \times 10^{-4}$ \\
\hline $\mathbf{a}_{18}=$ & 0.079 & $\mathbf{a}_{18}=$ & 0.01268 & $a_{18}=$ & $4.83372 \times 10^{-4}$ \\
\hline$a_{19}=$ & 0.0214 & $a_{19}=$ & -0.02040 & $a_{19}=$ & $3.85927 \times 10^{-4}$ \\
\hline$a_{20}=$ & $3.50228 \times 10^{-4}$ & $a_{20}=$ & $5.5514 \times 10^{-4}$ & $a_{20}=$ & $1.40104 \times 10^{-4}$ \\
\hline $\mathbf{a}_{21}=$ & 0.0108 & $\mathbf{a}_{21}=$ & 0.0132 & $\mathbf{a}_{21}=$ & 0.00108 \\
\hline $\mathbf{a}_{22}=$ & $7.55662 \times 10^{-5}$ & $\mathbf{a}_{22}=$ & $-1.43928 \times 10^{-4}$ & $a_{22}=$ & $-3.41598 \times 10^{-7}$ \\
\hline$a_{23}=$ & $1.40615 \times 10^{-4}$ & $\mathbf{a}_{23}=$ & $-7.15422 \times 10^{-4}$ & $\mathbf{a}_{23}=$ & $-5.06808 \times 10^{-4}$ \\
\hline $\mathbf{a}_{24}=$ & $8.33111 \times 10^{-6}$ & $\mathbf{a}_{24}=$ & $1.64481 \times 10^{-4}$ & $\mathbf{a}_{24}=$ & $4.48305 \times 10^{-5}$ \\
\hline $\mathbf{a}_{25}=$ & -0.01212 & $\mathbf{a}_{25}=$ & 0.04226 & $\mathbf{a}_{25}=$ & $-6.47156 \times 10^{-4}$ \\
\hline
\end{tabular}




\section{Conclusion}

We developed a Ronchi tester which has the capability to check the quality of any converging symmetrical system by calculating the transverse and the wavefront aberration in an expansion series of Zernike polynomials. We improved the source of illumination, being able to change the intensity of any LED by changing the voltage and wavelength of the LED. Also we developed the phase stepping technique. We find $1 \lambda$ resolution for our device, which can be obtained for any converging mirror but it could be extended to any symmetrical system. We tested optical systems such as mirrors made from aluminum alloy 6061, mirrors made with coated or non-coated silica glass and the biconvex and plane convex lenses with entrance and exit pupils on the plane surface. We used a modified version of Ghiglia's algorithm for circular domain shapes, which reduces 102 times the time consumed in the calculations. We also checked the optical quality of several microscope and telescope objectives with different $f$-numbers, with our device making it be easy and fast to do.

\section{Acknowledgements}

This work was financially supported by CONACyT, Mexico, under project \#J50614. Thanks to Gabriel Gordiano, Monica Pérez, Luis Sastre, Marco Antonio de Jesus, Pedro Tecuatl, Emanuel Carlock, Alberto Cordero and the reviewers for your help to improve this paper. GSO agradece al SNI. 